\title{
A Distinguished Retentive Memory Using Polyethylene Glycol Electrolyte Solvent for Viologen Modified Titania Electrochromic Device
}

\author{
Yoshitaka SANEHIRA, ${ }^{*}$ Satoshi UChIDA, ${ }^{\text {b }}$ Takaya KuBO, ${ }^{\mathrm{b}}$ and Hiroshi SegawA ${ }^{\mathrm{b}}$
}

\author{
${ }^{a}$ Graduate School of Environmental Studies, Tohoku University (Sendai 980-8579, Japan) \\ ${ }^{b}$ Research Center for Advanced Science and Technology (RCAST), The University of Tokyo (Tokyo 153-8904, \\ Japan)
}

Received August 27, 2007 ; Accepted December 10, 2007

\begin{abstract}
The electrochromic cell, which consisted of transparent conductive substrate, electrolyte and nano-crystalline titania thin film modified with viologen was assembled. The solvent dependency on kinetic of these cells was mainly investigated. When polyethylene glycol (PEG) was used as a solvent, the cell clearly indicated fast electrochromic reaction under an applied potential of $3.0 \mathrm{~V}$. The polyethylene glycol (molecular weight: 200) electrolyte was showed the best performance for keeping a strong coloration state for more than one month without any applied potential, thus making it an attractive candidate for paper-like displays with low energy consumptions.
\end{abstract}

Key Words : Electrochromic, Display, Viologen, Titania Electrode, Electrolyte Solvent

\section{Introduction}

The electrochemical and photochemical properties of various $1,1^{\prime}$-(R,R')-4,4'-bipyridinium (viologen) compounds have been studied. They have been used as mediators of photocatalytic and enzymatic reactions, because they act as good electron acceptors. ${ }^{1,2)}$ The viologen dication is easily reduced to its radical cation and is further reduced wherein the radical cation present a strong coloration with a high molecular absorption coefficient based on the optical charge transfer of the bipyridinium rings. ${ }^{3-5}$ Therefore, many viologen derivatives have been synthesized to customize the energy level, absorbance and redox potential according to the desired application. ${ }^{8,9)}$

A viologen redox couple had been also applied to the electrochromic display, which consists of simply an electrochemical cell structure and is driven by an applied voltage. ${ }^{5,6)}$ Advantages of electrochromic displays include inherent memory, low switching voltage, low mean power, and good contrast. Furthermore these type of displays are still in development by the introduction of a thin film electrode of metal oxide semiconductor with high porosity and suitable conduction band. ${ }^{7-11)}$ The mesoporous semiconductor film, mainly the titania electrode, functions as the support for a large amount of chromophores and also as the electron transfer channel to the anchored viologen molecules. Many researchers studied about a semiconductor electrode of this type of display and focused their attention on the absorption spectrum, rapid response, and cyclic characteristic of the electrode, but there is little mention of memory lifetime. This indicates that the conventional electrochromic device is easily discolored after releasing voltage.

In this paper, we investigated about the influence of the electrolyte solvent for charge transfer kinetics between viologen and the titania electrode, and could obtain a distinguished retention time of a colored state without applying any electric power.

\section{Experimental Section}

Bis(2-phosphonylethyl)-4,4'-biphyridinium dichloride (Viologen) was prepared according to literature. ${ }^{7-9)}$ The nano size crystalline Titania powder (P25, Average diameter: $21 \mathrm{~nm}$, BET surface area: $50 \mathrm{~m} \mathrm{~g}^{-1}$, Nippon Aerosil Co. Ltd.) was used as an electrode. All other chemicals were purchased and were of analytical reagent grade.

Titania electrode of dye-sensitized solar cells is applied as the support for the chromophores. Titania powder (1.0 $\mathrm{g})$, nitric acid ( $\mathrm{pH} 0.72,2.3 \mathrm{~g})$, acetylacetone $(0.1 \mathrm{~g})$, polyethylene glycol (MW: 500,000, $0.05 \mathrm{~g}$ ), and surfactant (10 wt $\%$ Triton X-100 aqueous solution, $0.05 \mathrm{~g}$ ) were placed in a Teflon ${ }^{\circledR}$ tube $(30 \mathrm{ml})$ with zirconia balls $(\phi=1 \mathrm{~mm})$ and the mixture was shaken with a paint mixer (500 $\mathrm{rpm}$, more than $10 \mathrm{~h}$ ). The transparent conductive glass substrate (FTO: F-doped tin oxide, $10 \Omega \mathrm{cm}^{-2}$, Nippon Sheet Glass Co., Ltd.) was used as both working and counter electrode. The paste was spread on the FTO/glass substrate (Film thickness: $10 \mu \mathrm{m}$, Working area: $20 \mathrm{~mm} \times 20 \mathrm{~mm}$ ) and it was annealed at $500{ }^{\circ} \mathrm{C}$ for $30 \mathrm{~min}$. The sintered electrode was immersed in a 10 $\mathrm{mM}$ viologen/ethanol solution for one night to absorb the saturated amount of dye on the titania surface, rinsed with ethanol, and dried before use.

The titania electrode and the counter electrode were sealed with a resin sheet (HIMILAN, $20 \mu \mathrm{m}$, Du PontMitsui Polychemicals Co. Ltd.) and the cell gap was filled with electrolyte $\left(0.05 \mathrm{M} \mathrm{LiClO}_{4}\right)$. A $\gamma$-butyrolactone (GBL), polyethylene glycol ( $\mathrm{PEG}_{200}, \mathrm{MW}: 200$ and $\mathrm{PEG}_{600}, \mathrm{MW}$ : $600)$, 3-methylpropionitrile (3MePN) and 1-ethyl-3-methylimidazolium bis(trifluoro-methylsulfonyl)imide (EMImTFSI) were used as electrolyte solvents.

Current and spectral behavior of the electrochromic cell depended on the potential sweep were simultaneous- 
ly observed. Potentio-galvanostat (HSV-100, Hokuto Denko Co., Ltd.) and UV-Vis spectrometer (UV-2450, Shimadzu Co., Ltd.) were used to record the current-voltage data of the electrochromic reaction and to measure the absorbance of the colored cell under applied potential, respectively.

\section{Result and Discussion}

The colored/bleached state image and the absorption spectra of the electrochromic cell is shown in Fig. 1. The cell consists of a nano-crystalline titania film electrode modified with viologen and GBL electrolyte. The titania electrode is connected to the cathode, and the counter electrode is jointed to the anode of the electric power source. The cells were uniformly deep blue and exhibited the strongest coloration under a supplied voltage of $3.0 \mathrm{~V}$ and then bleached under opposite bias, which demanded a higher potential than previous reports. ${ }^{7-10)}$ In the visible range, the absorption spectrum of the bleached state corresponds to that of titania, however, it exhibit a broad strong absorption centered at around $550 \mathrm{~nm}$ in the colored state. Since the viologen chemically bonded and filled the titania surface, they never desorbed to the electrolyte for all states. ${ }^{7-10)}$ A titania electrode works not only as the support for abundant viologen but also as the diode that controls the redox reaction to only one direction according to the applied voltage. This suggests that the viologen radical cation is generated by the injection of an electron from the titania electrode. Moreover, the absorption spectrum is consistent in all electrolyte solvents in this work. Hereafter, we evaluate the absorbance at $550 \mathrm{~nm}$ which has a high visibility with strong contrast in the following examinations.

In Fig. 2, the absorbance change at $550 \mathrm{~nm}$ depended on the applied potential sweep. At first, the applied bias was gradually increased from $0.0 \mathrm{~V}$ to $3.0 \mathrm{~V}$ to lead to the coloration of the chromophores, decreased to $-3.0 \mathrm{~V}$ to bleach the cell, and then turned to $0.0 \mathrm{~V}$. The whole operation was carried out at the rate of $50 \mathrm{mV} \mathrm{s}^{-1}$. Although all cells indicated a maximum absorbance at $3.0 \mathrm{~V}$ of applied bias, the response of maximum absorption intensity is different from few electrolyte solvents. The colorations of GBL and two PEG electrolytes cells started at a lower bias and exhibited stronger absorptions than those of $3 \mathrm{MePN}$ and EMIm-TFSI. On the other hand, the bleaching process in PEG electrolytes occurred at lower potential than the other solvents.

The reduction/oxidation current according to the coloration/discoloration of viologen were simultaneously observed and were found to behave similarly in all electrolyte solutions. However, the total quantity of electricity between the forward and back reactions was not always equal in all cells. The two PEG electrolytes exhibit close agreement with the total quantity of electricity on the reduction/oxidation current $\left(\mathrm{PEG}_{200}: 0.82 \mathrm{mC} \mathrm{cm}^{-2} / 0.79\right.$ $\mathrm{mC} \mathrm{cm}{ }^{-2}, \mathrm{PEG}_{600}: 0.63 \mathrm{mC} \mathrm{cm}^{-2} / 0.63 \mathrm{mC} \mathrm{cm}^{-2}$ ), while the others indicate a decrease in and peak broadening of the current on the oxidation reaction of viologen. The cells with GBL and PEG electrolytes both had strong absorbances, but the charge of reduction reaction in GBL

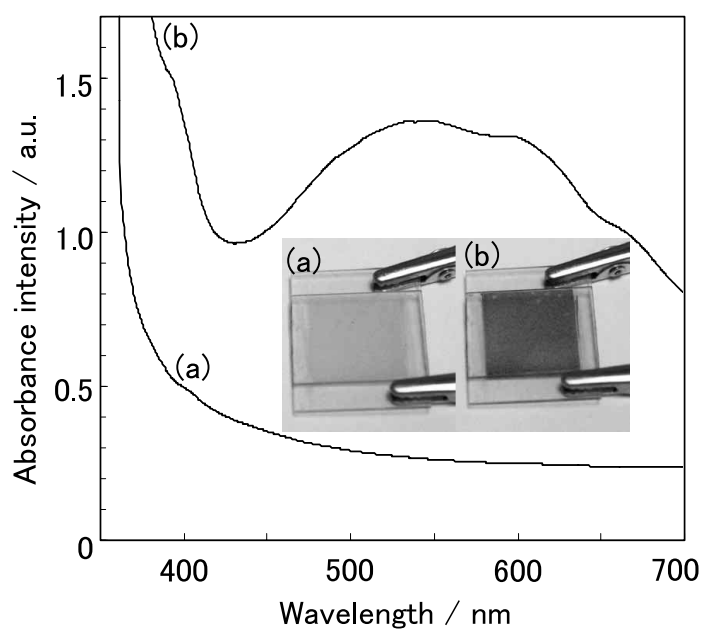

Fig. 1 Absorbance spectrum of (a) decolorized and (b) colored state of titania film impregnated with viologen.

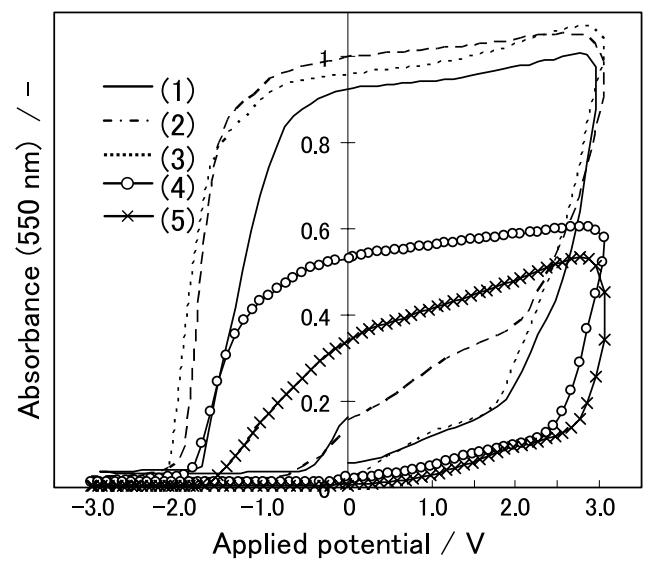

Fig. 2 Variation of (a) abosorption intensity at $550 \mathrm{~nm}$ versus applied potential between electrodes, using (1) GBL, (2) PEG (MW: 200), (3) PEG (MW: 600), (4) 3MePN, and (5) EMIm-TFSI as an electrolyte solvent.

electrolyte was higher than that of the PEG electrolytes $\left(1.77 \mathrm{mC} \mathrm{cm} \mathrm{cm}^{-2} / 0.75 \mathrm{mC} \mathrm{cm}{ }^{-2}\right)$. The $3 \mathrm{MePN}$ and EMImTFSI electrolytes showed lower absorbances in their reduction current (3MePN: $1.19 \mathrm{mC} \mathrm{cm}-2 / 0.44 \mathrm{mC} \mathrm{cm}^{-2}$, EMIm-TFSI: $0.87 \mathrm{mC} \mathrm{cm}-2 / 0.43 \mathrm{mC} \mathrm{cm}^{-2}$ ). Not all those quantities of charge on reduction were in agreement with the absorbance, but those of oxidation were proportional to the absorbance of each cell. This suggests that the efficiency of the coloration reaction is high in the PEG electrolytes. It can be postulated that a lot of charge could have leaked from electron transfer on the interface of the electrode and is highly influenced by the electrolyte solvent.

The coloration in the PEG and GBL electrolyte were demonstrated in a lower applied potential than that of the 3MePN and EMIm-TFSI electrolyte in Fig. 2. The electrochromic reaction of cells are attributed to the reduction and oxidation of viologen. ${ }^{3-5)}$ The reduction arises from the electron injection to the viologen dication via the conduction band of titania, while the oxidation is attributed to the back electron transfer reaction. Since 

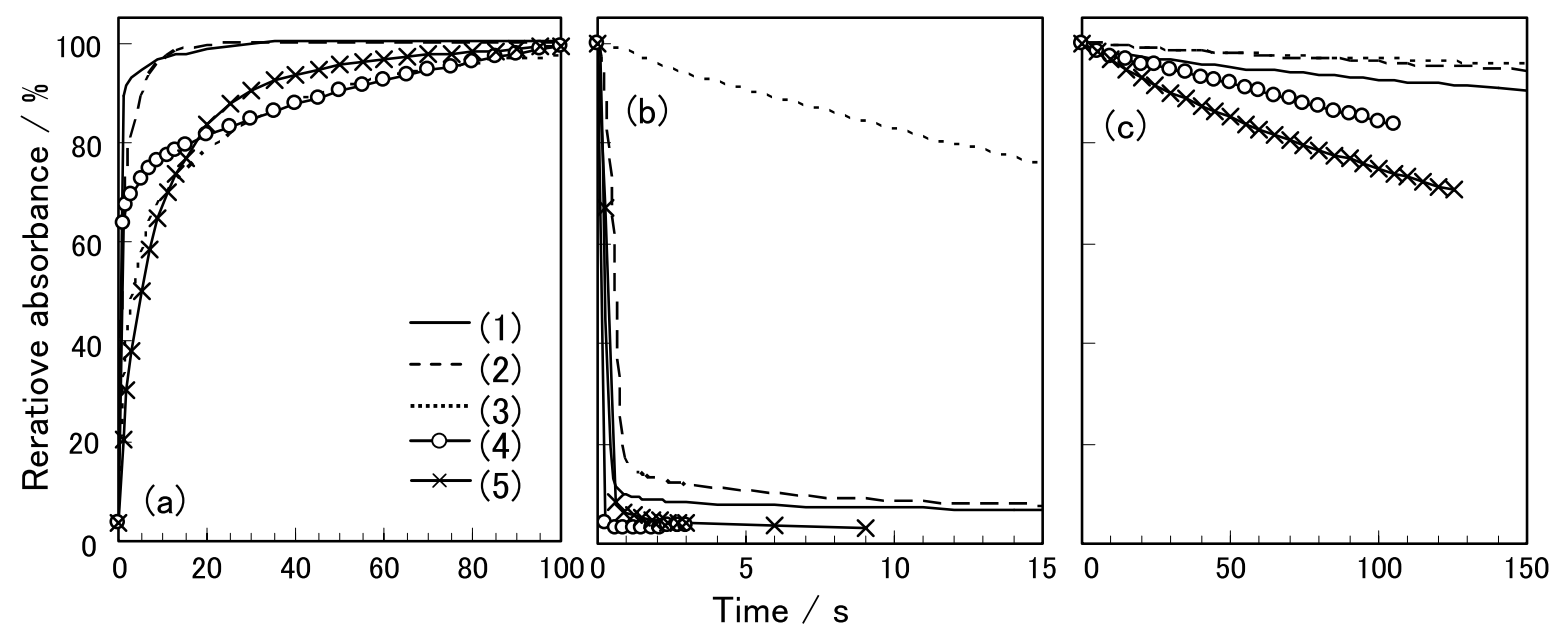

Fig. 3 The transient responses of (a) coloration (switching $0.0 \mathrm{~V}$ to $3.0 \mathrm{~V}$ ), (b) discoloration (switchng $3.0 \mathrm{~V}$ to $-3.0 \mathrm{~V}$ ) stage and (c) the retation time of memory (switching $3.0 \mathrm{~V}$ to $0.0 \mathrm{~V}$ ). Lines are correspond to (1) GBL, (2) PEG (MW: 200), (3) PEG (MW: 600), (4) 3MePN, and (5) EMIm-TFSI, respectively.

the redox energy level of viologen and the titania conduction band edge are relatively close to each other resulting to a small energy gap, such reversible reactions are enabled even with only a slight applied bias. ${ }^{9-11)}$ On the other hand, it is known that the photon to electron conversion efficiency is influenced by the electrolyte solvent in dye-sensitized solar cells. ${ }^{12)}$ It is explained that the electron accepting and/or donating properties of the solvent cause a shift in the conduction band edge of titania, thus, altering the injection efficiency of photoinduced electrons of the sensitizer. It is possible that the similar band edge shift of the titania conduction level would inhibit a smooth electron transfer onto the viologen/titania interface thereby increasing the bias potential, which is necessary for viologen redox reactions. Furthermore, charge recombination reaction with ion species that diffused from the counter electrode side can be regarded as another channel of charge leakage. ${ }^{7)}$ It will promote the bleaching of viologen even if enough potential is applied in low viscosity solvent such as GBL and $3 \mathrm{MePN}$. Thus, it can also be assumed that the viscosity of solvent which influences ionic conductivity is an important parameter as well. Such properties of electrolyte solvents affect not only the coloration/discoloration efficiency but also the response rate when constant bias was applied.

The transient response of absorbance at $550 \mathrm{~nm}$ was investigated under constant bias and is shown in Fig. 3. The cell was supplied constant voltage $(3.0 \mathrm{~V})$ and was then replaced with the opposite voltage $(-3.0 \mathrm{~V})$ in order to observe the switching of coloration/discoloration states, respectively. The retention times of the colored state with the various electrolytes after switch off of the coloration voltage were also examined. The obtained result is normalized by the maximum absorbance of the almost completely colored state. $\mathrm{GBL}$ and $\mathrm{PEG}_{200}$ electrolytes reached the steady state faster than the others in the coloration phase (Fig. 3(a)), but all of them were bleached immediately except for $\mathrm{PEG}_{600}$ (Fig. 3(b)). In the examination of the retention time of the colored state, two PEG electrolytes especially maintained a strong

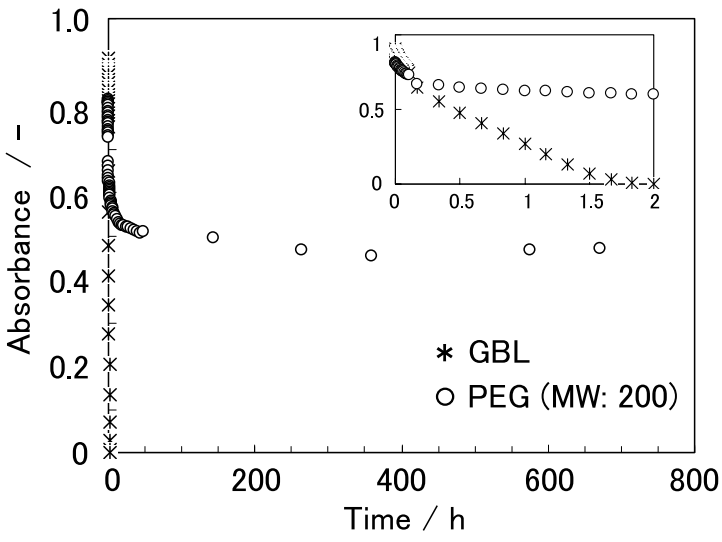

Fig. 4 Long term memorability of electrochromic cell udner using BGL and PEG (MW: 200) electrolyte.

absorption for a long amount of time, while 3MePN and EMIm-TFSI electrolytes were rapidly bleached. The oxidation of the viologen cation can be promoted by ion species that diffused from the electrolyte. ${ }^{7)}$ This suggests that the charge dispersion of the viologen radical cation is suppressed in the PEG electrolyte with low ionic conductivity, but, on the other hand, it is liable to occur in 3MePN and EMIm-FTSI electrolyte even if positive bias is applied. It can be assumed that not only the electron accepting/donating property but also the viscosity of the solvent surrounding the electrode greatly affects charge transfer.

The long-term stability of the display/non-display state without electric means is an important property in display media, since it is allows for the decrease in energy consumption. The durations of colored viologen in GBL and $\mathrm{PEG}_{200}$ electrolyte, which can switch rapidly between the colored/discolored states were evaluated from the decay curves of the absorbance at $550 \mathrm{~nm}$. In Fig. 4, the absorbance of the GBL electrolyte cell completely bleached in less than $2 \mathrm{~h}$ after the voltage supply was stopped. However, the PEG electrolyte cell kept its strong absorption for more than one month, about 400 
times longer than that of the GBL cell. We also found that the decay curves consist of an exponential steep slope within a several minute period followed by a slow quenching in both electrolytes. The former is assumed to be attributed to the offset of charge on the dye cation as well as the electric double layer capacitor that arose from an applied potential leading to coloration. Because of the similar absorbance and oxidation current that were obtained from the coloration examination in Fig. 2, it can be estimated that cells can hold an equivalent amount of charge. This is also supported by the corresponding rapid absorbance decay at less than -2 orders of magnitude hour in Fig. 4. On the other hand, the latter can be attributed to the charge dispersion of oxidizing agents diffused from the electrolyte. Since such diffusion is governed not by coulomb interaction but by the viscosity of the solvent, it can be considered that the suppression of mass diffusion is an important factor for long period retention of the viologen radical cation.

The $\mathrm{PEG}_{200}$ electrolyte cell exhibited a moderately sensitive responsiveness to bias switching and displayed a distinguished long retention of coloration without an applied electric power source. This leads to the conclusion that the viscosity of the electrolyte solvent is an important factor for not only rate-determining step but also the long-lifetime of the colored state. However, to develop a more rapid response and a longer period stability of the colored/discolored state, a detailed study of viologen oxidation in various electrolytes is still necessary for the construction of better electrochromic displays.

\section{Reference}

1) D. L. Erbes and R. H. Burris, Biochimica Biophisca Acta, 525, 45 (1978).

2) T. Nakato, K. Kuroda, and C. Kato, Chem. Mater., 4, 128 (1992).

3) H. T. van Dam and J. J. Ponjeé, J. Electrochem. Soc., 121, 1555 (1974).

4) I. F. Chang, B. L. Gilbert, and T. I. Sun, J. Electrochem. Soc., 122, 955 (1975).

5) T. Kawata, M. Yamamoto, M. Yamana, M. Tajima, and T. Nakano, Jpn. J. Appl. Phys., 14, 725 (1975).

6) C. J. Schoot, J. J. Ponjee, H. T. van Dam, R. A. Doorn, and P. T. Bolwijn, Appl. Phys. Lett., 23, 64 (1973).

7) R. Cinnsealach, G. Boschloo, S. N. Rao, and D. Fitzmaurice, Sol. Energy Mater. Sol. Cells, 55, 215 (1998).

8) R. Cinnsealach, G. Boschloo, S. N. Rao, and D. Fitzmaurice, Sol. Energy Mater. Sol. Cells, 57, 107 (1999).

9) D. Cummins, G. Boschloo, M. Ryan, D. Corr, S. N. Rao, and D. Fitzmaurice, J. Phys. Chem. B, 104, 11449 (2000).

10) P. Bonhôte, E. Gogniat, F. Campus, L. Walder, and M. Grätzel, Displays, 20, 137 (1999).

11) S. Y. Choi, M. Mamak, N. Coombs, N. Chopra, and G. A. Ozin, Nano Lett., 4, 1231 (2004).

12) K. Hara, T. Horiguchi, T. Kinoshita, K. Sayama, and H. Arakawa, Sol. Energy Mater. Sol. Cells, 70, 151 (2001). 